villes citées comme exemple n'ayant été effectuée que pour l'éclairage public seulement, et par suite n'étant pas totale;

Mais considérant que la disposition précitée à l'article $2, \S 3$ du cahier des charges ne peut être entendue comme imposant à la Ville requérante l'obligation de justifier que les villes, citées par elle comme exemple, aient remplacé un système exclusif d'éclairage au gaz par un nouveau système d'éclairage exclusif; que d'après le sens attribué à la disposition, il suffit que ce nouveau mode soit appliqué dans ces villes concurremment avec l'éclairage au gaz dans la totalité du périmètre desservi par ce dernier mode d'éclairage ;

Considérant que l'état de l'instraction ne permet pas d'apprécier dès à présent :

I) Si dans les villes indiquées par la Ville de Carpentras le nouveau mode d'éclairage adopté pour l'éclairage public s'applique danstout le périmètre desservi par les Compagnies du Gaz;

2) Si dans la Ville de Carpentras, l'installation de l'éclairage par l'électricité dans les conditions du projet proposé à la Ville par le Sieur Rivoire serait plus avantageux et plus économique pour elle que l'éclairage au gaz actuel; qu'il y a lieu dès lors d'annuler l'arrêté attaqué et d'ordonner une expertise à l'effet de rechercher si ces deux conditions sont remplies;

\section{DÉCIDE :}

Article r. - L'arrêté ci-dessus visé du Conseil de Préfecture du Département de Vaucluse, en date du 19 avril Igo2, est annulé.

Art. 2. - Avant de faire droit au fond, il sera, par un expert si les parties s'entendent pour la désignation d'un seul expert, sinon par trois experts dont lun sera désigné par la Ville de Carpentras, le second par la Compagnie d'Eclairage au Gaz et le troisième par le Président de la Section du Contentieux,procédé à une expertise.

Le ou les experts prêteront serment entre les mains, soit du Secrétaire du Contentieux du Conseil d'Etat, soit du Vice-Président du Conseil de Préfecture du Département de Vaucluse.

La mission du ou des experts consistera à rechercher:

I) $\mathrm{Si}$ dans les villes indiquées par la Ville requérante le nou. veau mode d'éclairage s'applique à tout le périmètre desservi par la Compagnie du Gaz;

2) Si l'établissement et l'exploitation de l'éclairage par l'électricité des voies publiques et des monuments municipaux dans les conditions du projet proposé à la Ville de Carpentras par le sieur Rivoire seraient pour elle plus avantageux et plus économique que l'éclairage actuel par le gaz.

Le ou les experts déposeront leur rapport au Secrétariat du Contentieux du Conseil d'Etat dans un délai de trois mois à dater de la prestation de serment.

Art. 3. - Les dépens sont réservés pour être supportés par la partie qui succombera en fin de cause.

\section{LE PETIT MOTEUR ÉLECTRIQUE}

Ainsi que les lecteurs de La Houille Blanche le savent dèjà (") la Socièlé d'Agriculture, Sciences et Industrie do Lyon a organisé, du 1 er au 25 août demier, une Lxposition du « petit moteur électrique dans ses applications aux usages domestiques et à l'atelier familial s; et on ne peut que la féliciter de son intelligente initıative.

Il était en offet utile de montrer au public français. et particulièrement à celui de la région lyonnaise, leś avantages multiples qu'il pouvait retirer de l'emploi du petit moteur électrique, - et aux constructeurs, qu'll était temps qu'ils songent à faire utiliser plus complètement dans le ménage, aussi bien qu'au petit atelier, par des appareils appropriés, celte énergie électrique si facilement et si abondamment distribuée, ce qui doit se traduire forcément par un champ d'action plus vaste pour leur industrie.

L'étranger nous a déjà précédé dans cette voie, l'Angleterre, et l'Allemagne surtout, nous ont distancé de beaucoup, tant pour l'utilisation que pour la production. C'est

() Voir La Houille Blanche, no de mat 1906 par milliers que, dans Berlin, on compte les potits moteurs appliqués soit à la commande individuelle dans les grandes manufactures, soit aux outils des petits façonniers, soit, entin, aux appareils domestiques de nettoyage et d'ali. mentation.

Le petit moteur électrique se prête très bien aux appli. cations les plus diverses; sa forme, qui est variable suivant les besoins, son peu de volume, sa facilité d'alimen. tation par des organes souples et d'encombrement réduit, l'absence de résidus de fonctionnement, sa commodité d'entretien, sont autant de facteurs qui plaident pour son entière vulgarisation. Les applications, de leur côté, peuvent être aussi nombreuses que variées: dans le ménare, la préparation des aliments, le nettoyage, la ventilation; dans les petils ateliers, le tissage, le dévidage, la couture, lo découpage, le perçage, le burinage, etc. Ce sont là autant de besognes auxquelles on peut asservir le petit moteur, ct cela pour le grand soulagement de l'ouvrier qui les fait actuellement, en lui évitant ainsi le pénible labeur corporel auquel il est attelé, et ne lui laissant que la direction de l'outil, c'est-à-dire le travail intelligent de l'esprit. Le potit moteur a une qualité de plus à son actif, qualité précieuse en notre siècle, il est égalitaire et démocratique.

La Société d'Agriculture, Sciences et Industrie avai donc, on le voit, choisi un sujet intéressar.t.

Nous ne pouvons décrire ici toutes les applications qui étaient présentées à ce concours par les nombreuses maisons qui y ont pris part; aussi nous bornerons nous à donner quelques indications sur les appareils les plus inté. ressants qui ont été l'objet de récompenses.

I.e jury était ainsi composé : Président: Desuuzeur, directeur de l'Association lyonnaise des propriétaires à vapeur membres: MM. Godinot, vice président de la Société; Cốtr président de la Commission du Concours, Magenties, Rodet, Courbier, Charlet, Heilmann. La maison Gindre, Duchavany et Cie, représentée par M. LIMB, et la Société de constructions électriques ot Cadiot, représentée par M. Dukard, étaient hors concours et membres du jury.

Aspirateur de poussière. - Sous la forme d'un élégant petit meuble, l' “ aspirator" " est un appareil de nettoyage par le vide qui répond à toutes les exigences du confort ei de l'hygiène moderne. C'est-à dire qu'avec cet appareil on

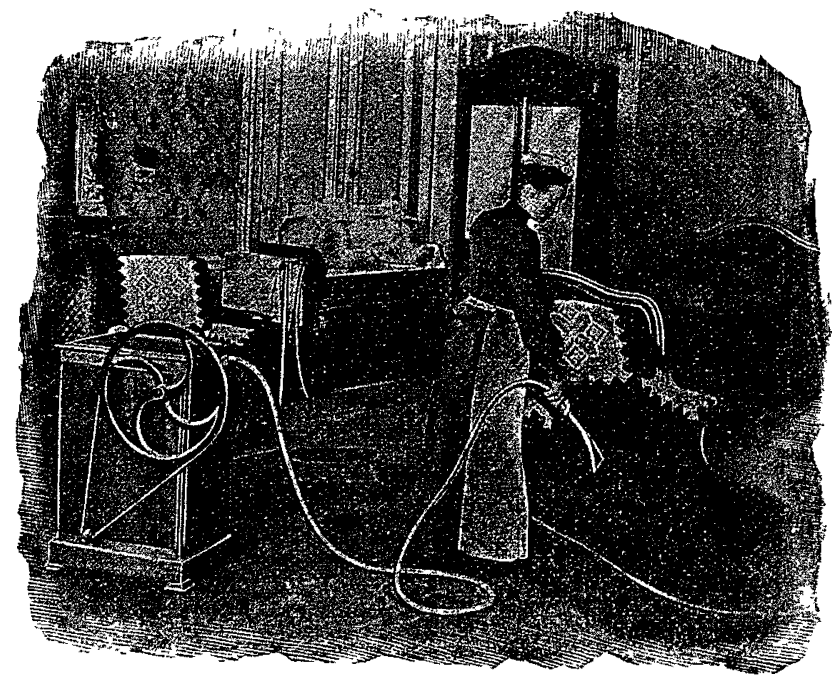

peut neltoyer partout, dans les recoins les plus inaccessibles comme sur les tentures les plus compliquées, et cela sans soulever la moindre poussière, sans déplacer le moindre microlje; au contraire, tout est absorbé, et peut être facilcment détruit.

Un simple petit moteur de $1 / 4$ de cheval actionne un jell de souffiets produisant une dépression à peu près constante, dépression cjui peut être utilisée à l'extrémité d'une tubulure flexible à laquelle on peut adapter des bouches de 
formes différentes suivant leur destination : bouches plates pour tapis et tentures, que l'on utilise ou bout d'un tube rigide, bouche à capiton, brosses à meubles, etc. Entre les soufflets et la tubulure, se trouve intercalé un réservoir mobile muni d'un filtre qui retient la poussière. Ce réservoir est muni d'un couvercle pour permettre la vidange des poussières iorsque le nettoyage est terminé.

On emploie industriellement cet appareil pour le nettoyage des casses d'imprimeric, sans avoir à déranger celles-ci; pour cela, on adapte au tube flexible une bouche speciale de forme carrée, dont l'irtérieur est muni d'une tolle métallique, et le raccord d'un orifice. Pour faire ce nettoyage, on pose la bouche sur les caractères, et, l'orifice du raccord étant obstrué par le doigt, l'aspiration se produit ct soulève les caractères, qui sont retenus par la toile métallique et sont débarrassés de lour poussière. Si l'on démasque lorifice, les caractères retombent à leur place. Notre imprimeur ne nettoie pas autrement ses caractères.

Cet appareil a obtenu un premier prix avec médaille de vermeil.

Brosse mécanique pour parquets. - Avant de terminer le chapitre du nettoyage, nous signalerons une brosseuse méranique de parquets présentée par M. Bruyére. Cet appareil se compose de trois brosses traversées par une pièce de fer. Les deux brosses extrêmes se déplacent en conlissant sur cette pièce de fer, alors que la brosse centrale, qui porte un petit moteur électrique, tourne constamment sur elle-même. Une tige de bois, munie d'une poignće, sert à promener la brosse, en mênıe temps qu'un fil souple assez long amène le courant électrique.'

Cet intéressant appareil a été gratifié d'un deuxième prix, avec médaille d'argent et prime de 100 francs.

Appareils de laboratoire. - M. MAURY, constructeur d'appareils de précision, à Lyon, avait exposé un certain nombre d'appareils de laboratoire, notamment:

Une étuve de dessication. - Cette étuve, entièrement métallique, est à double paroi isolante garnie de matière imputrescible. Sa porte est à coulisse verticale, et est équilibrée par deux contrepoids reliés à l'aide de câbles passant sur des poulies de renvoi. Elle est munie à l'intérieur d'un rayon en tôle porforée. Son chauffagre est obtenu à l'aide de radiateurs électriques placés à la partie inférieure, et elle est enveloppée par un carter en tôle qui la préserve de tout contact.

Cette étuve est à régulation automatique. Le régulateur se compose d'un interrupteur à cuvette de mercure, dont l'arc de fil de conducteur, qui plonge dans les cuvettes, est soulevé au moment voulu par un relai ainsi constitué: une dérivalion, prise sur le circuit de chauffe, circule dans deux bobines d'induction enroulées en série, et aux centres desquelles se trouvent deux noyaux de fer doux suspendus à un fléau mobile autour d'un axe. A l'un de ces noyaux de fer, est suspendu l'arc de contact qui déséquilibre la balance ainsi constituée. Le contact tend donc à exister continuelle- ment, mais sur la dérivation est intercalé un thermomètre à mercure qui plonge dans l'étuve. Le mercure de ce thermomètre forme un pôle, l'autre pôle étant constitué par une tige de platine réglable. Lorsque, sous l'influence de la température, le mercure vient en contact avec le platine, lo courant circule dans les bolsines, et la force portante du petit électroformé par le système de bobines vient rétabli. l'équilibre du fléau; celui-ci soulève alors l'arc métallique et rompt ainsi le contact.

Tentrifugeuse. - Les centrifugeuses, dont l'emploi so répand de plus en plus dans les laboratoires, servent soit en chimie analytique pour la réunion de certains précipités et la purification de certaines liqueurs, soit en chimie bio-

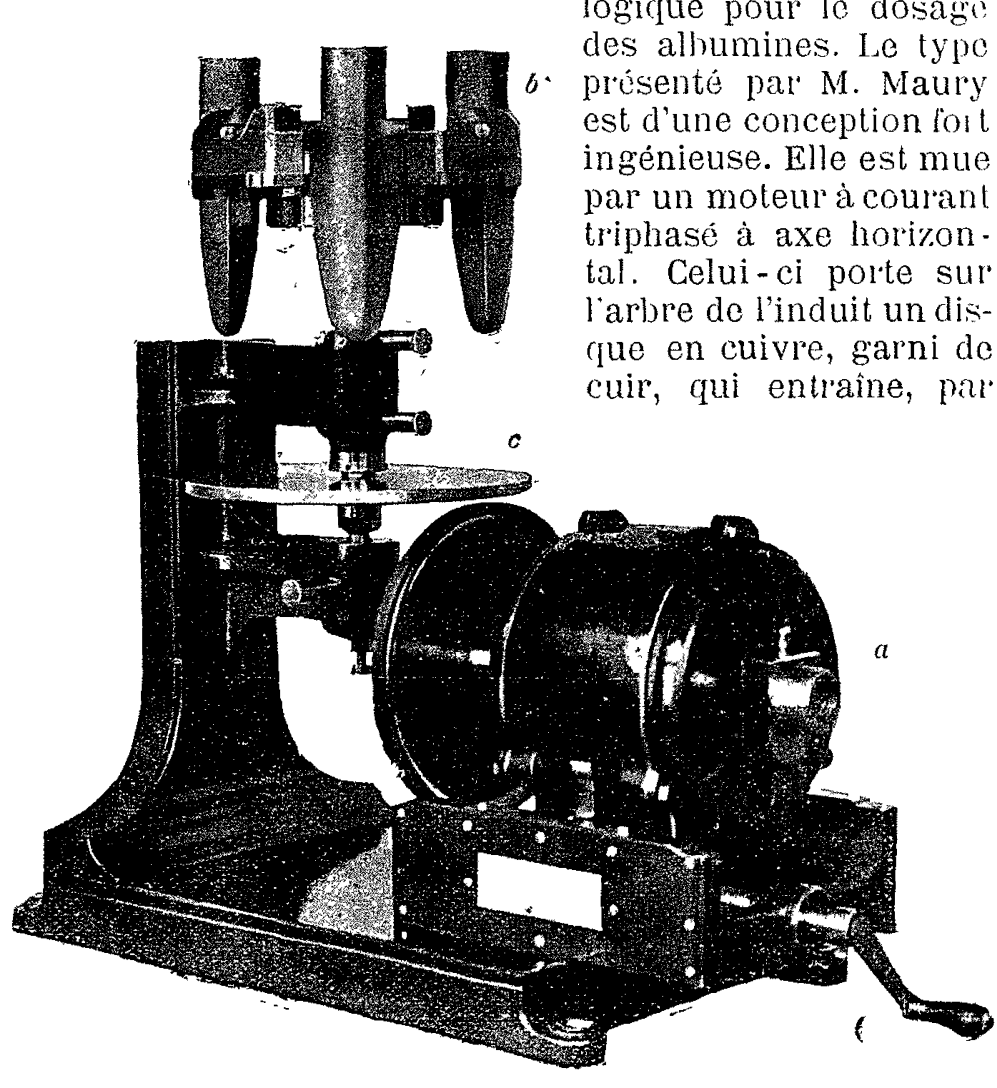

l'intermédiaire d'un autre plateau $c$, l'arlore varlical portant le porte-tube $b$.

Le moteur porte à son bâti un écrou qui engrène avcc une vis actionnée par la manivelle $d$, et qui sert à son déplacement sur deux rails fixés au bâti général de l'appareil. Le déplacement du moteur a pour brt : $1^{\circ}$ sa mise en marche, $2^{\circ}$ le lancement progressif de l'appareil. I c moleur démarre au promier quart de tour donné à la manivelle, car trois galets, portés par le moteur et formant bornec, viennent en contact avec trois lames d'arrivéc de courant fixées au bâti de la machine. A ce moment, l'entrainement du plateau est imparfait, car le disque d'entraînement étant en contact avec la périphérie du plateau, qui est bisautéc. il y a patinage. Si l'on continue à déplacer le moteur, l'adhćrence devient complète et la vitosse du portc-tube croit, puisque l'on rapproche le disque d'entraînement du centro du plateau entraîné. On a donc, à l'aide de ce dispositif, un cmbrayage absolument progressif, et une progression constante de la vitesse du porte-tuhes pendant sa mise en régime; les tubes centrifuges n'ont donc aucun choc' $a$ supporter.

Agitateur systine Arloing. - Cet appareil a été construit pour l'entretien des cultures de certains bacilles; il est dostiné à l'agitation régulière de ces liquides, afin de les conserver dans un état d'homogénéité parfait, et pour leur aération. Il est aclionné par un moteur monophasé d'une puissance de 7 kilogrammètres.

L'appareil agitateur proprement dit est supporté par 
quatre picds métalliques II se compose de deux systèmes de coulisses perpendiculaires, mobiles l'une sur l'autre; le sy'stème supérieur supporte le plateau agitateur. Une manivelle, montée sur un axe vertical, et portant un galet à son

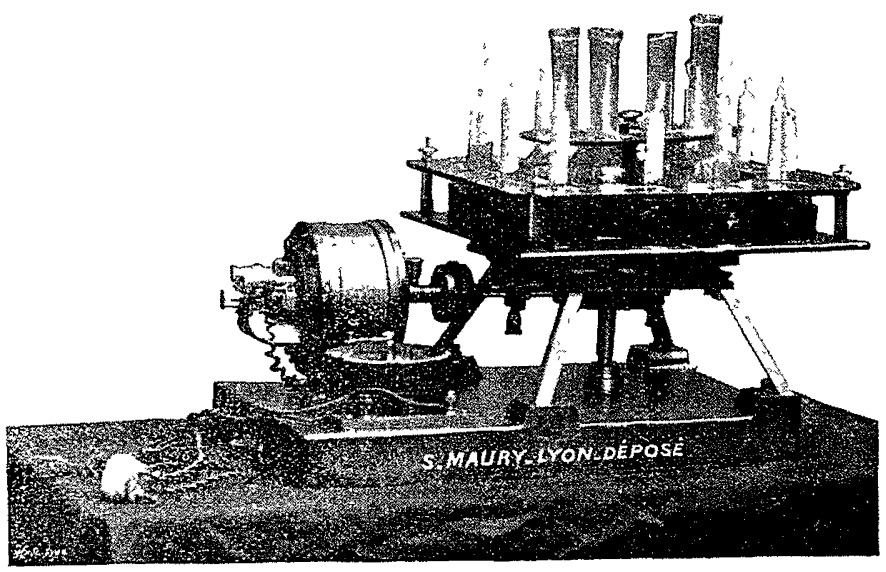

extrimiti, vient frapper alternativement les quatre côtés du carre forme par les coulisses, en donnant au plateau agitateur quatre mouvenents suivant deux directions perpendiculaires. Ces mouvements se traduisent par des chocs ful agitent les lijuides, tout en leur donnant un mouvement de rotation contre la paroi intérieure des flacons.

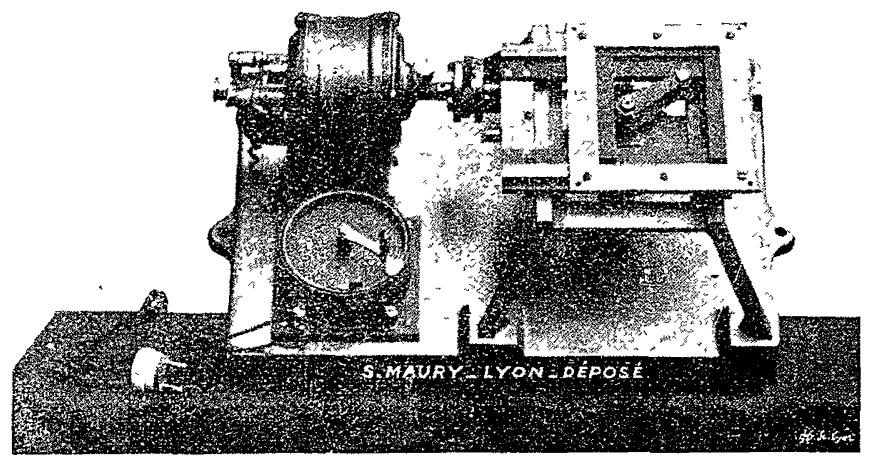

La transmission du mouvement du moteur à l'arbre de la manivelle se fail par une vis sans fin et un plateau à denture hélicoidale monté sur cet arlre. Pour amortir sur le moteur les chocs qui lui seraient nuisıbles, il existe entre l'arbı'c du moteur et l'arbre de la vis sans fill un accoupleincnt élastıque à anneaux de caoutchouc.

Apparcil enregistreur. - Lappareil présenté emploie un petit moteur a courant continu d'une puissance de deux kilograminètres environ, et d'une grande régularité. Il se compose d'un cylindre vertical à côté duquel se trouve une tige, sư laquelle on peut déplacer l'appareil porte style à laide d'une crémallère. La transmission du mouvement du moteur au cylindre se rait à l'aide d'une vis salıs fin et d'un plateau denté sur l'axe duquel se trouve un disque d'entrainement; le plateau entrainé est sur l'axe même du cylindro enregistreur, sur lequel il peut être déplacé pour permettre les variations de vitesse. Pour la commodité de l'emploi de l'appareil, lo constructeur l'a muni dun délsrayage constitué par un tambour creux, sur la paroi intérieure duquel frotle un galet entraineur monté sur l'axe du moteur. I.e moteur peut pivoter autour diun axe verlical sur lequel il est fixé, un ressort mántiont en contact constant le galet contre le tambour. Lorsque l'on veut alrêtor mstantanément le cylindre pour faire une observation, il sulfit de farre tourner à la main le moteur sur luimême de mamère à décoller le galet, et de fl'ciner simultanément sur le tarnbour avec le doigt. Le cylindre enregistreur peut fonctionner horizontalement ou verticalement, pour cela, son bâti est fixć au bâtı géuéral de la machine par une alticulation à plateau dont l'axe de rotation coincide avec laxe du plateau d'entrainement.
A l'ensemble de ces appareils a été attribuée une médaille de vermeil (rícompense spéciale offerte par l'Association lyonnaise des propriétaires à vapelir,.

Application du petit moteur à la Machine à coudre. La Maison Gelin, qui s'est fait une spécialité du pelit motsur pour machine à coudre, exposait plusieurs modèles intéressants d'adaplation à des machines à coudre et $\dot{a}$ broder.

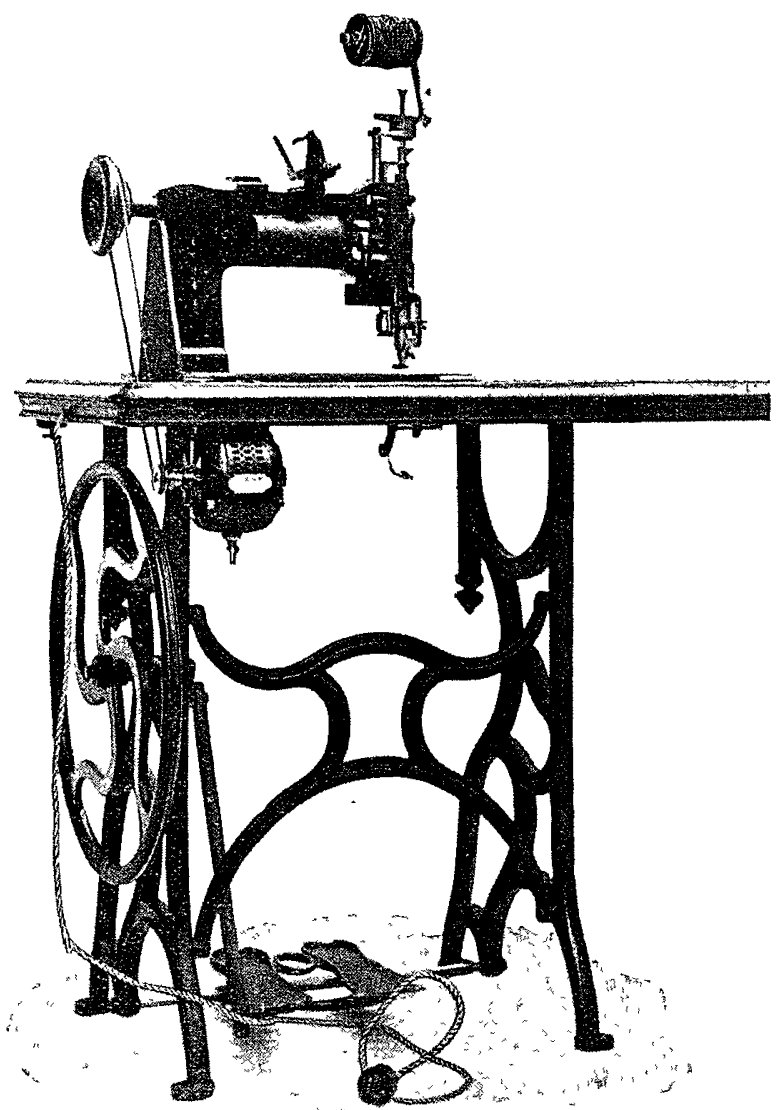

Dans un premier sy stème, le moteur est suspendu sous la tablette de la machine par une charnière dont l'axe est sur le côté du bâti. Comme son poids tend à le rejeter à l'extérieur, il forme donc tendeur de courroie automatigue; la tension peut encore être augmentée à l'aide d'une tige commandée par la pédale, dont l'effort vient s'ajouter ail poids du moteur. Pour la machine à broder, le moteur cuspendu de cette façon s'arrête chaque fois que l'on débraye la machine. A cet effet, un interrupteur est combiné avec la poignée de débrayage. Un autre système comporle un rhéostat de démarrage commandé par la pédale.

M. Gelin s'est vu décerner un deuxième prix avec médaille d’argent.

Pétrin mécanique. - Ce pétrin mécanique, présenté par $M$. Berutti, est un instrument dont le public consommateur aimerait beaucoup voir se généraliser l'emploi, car on y gagnerait certainement beaucoup en propreté. L'ouvrier boulanger lui-même n'en serait pas fâché, car il ne serait plus attelé au clur labeur qu'il est actuellement obligé de faire.

Ce pétrin est d'une simplicité de mécanisme et d'une robustesse de construction qui conviemnent fort bien $\dot{a}$ l'usage qu'il e-t appelé à remplir, et aux mains qui le manieront. Il emploie un moteur de 2 chevaux 1/2, à courant alternatif triphasé. Le pétrin par lui-même se compose d'un baquet légèrement tronconique, dont le plus grand diamètre est de un mètre, et qui est monté sur un axe vertical autour duquel il peut pivoter. Il possède un moyeu conique en bois dont l'inclinaison est la même que celle de 
la paroi du baquet. Entre le moyeu et la paroi du baquet se meut une palette montée sur un arbre vertical; cet arbre porte un engrenage horizontal à denture hélicoidale commandé par une vis sans fin montée sur l'arbre du moteur. Celui-ci, l'axe vertical de la palette, et l'axe du baquet luimême, sont portés par un bâti général en fonte. A vide, le

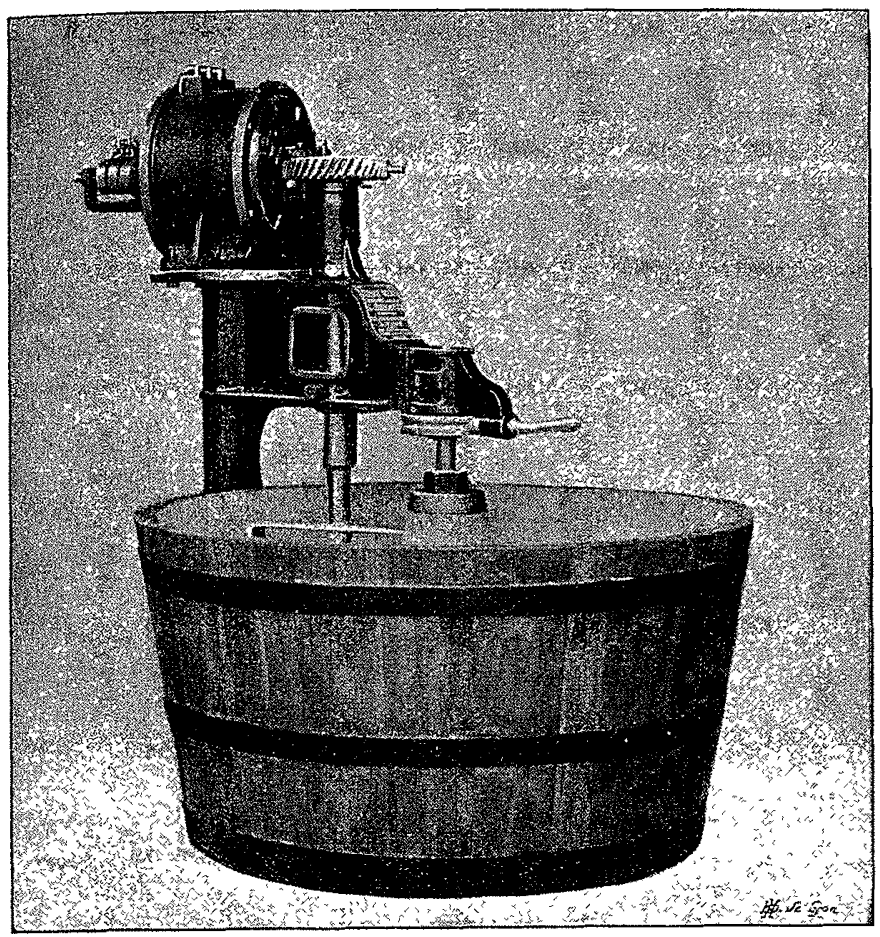

baquet est immobile, et ce n'est que lorsqu'il contient la pàte que son entraînement se produit. Le brassage se fait alors dans tout l'intérieur du baquet. Il sulfit d'une marche de 10 à 15 minutes pour pétrir $125 \mathrm{kgs}$. de pain.

Cet appareil s'est également vu altribuer un deuxième prix et une médaille d'argent.

Hache-viande. - Dans le stand de l'alimentation, M. DuCREUX exposait encore un hache viande â l'usage des bouchers et charcutiers. Il se compose d'un tube dans lequel tourne une vis. Celle-ci déchiquète la viande et la refoule à l'extrémité du tube où elle est obligée de passer à traver's un treillis métallique résistant, à maille assez serrée.

Un troisième prix, avec médaille de bronze, a été décerné à cet appareil.

Machine à dévider. - M. Lespinasse exposait une machine à dévider mue par un petit moteur électrique, de 1/6 de cheval environ, construit par la Société alsacienne de constructions mécaniques. Cet ensemble, qui a particulièrement intéressé les canuts de la région lyonnaise, s'est vu décerner par le jury un deuxième prix avec médaille d'argent.

Machine à imprimer. - Même récompense a été attribuée à M. RuA qui exposait une petite machine à imprimer les enveloppes, en-tètes de lettres, cartes de visites et de réclames.

\footnotetext{
Appareil à stériliser leau par lozone. - Cet appareil tout nouveau, et très intéressant, était présenté par la Compagnie pour la Fabrication des Compteurs et matériel d'usine à gaz. Il se compose d'un générateur d'ozone et d'un stérilisateur proprement dit. Le générateur d'ozone est constitué par une caisse métallique non hermétique, à l'intérieur de laquelle se trouve une bobine dinduction, qui reçoit sur son plinaire le courant du secteur s'il est alternatif; les deux bornes du secondaire sont en communication avec celle dun condensateur à lame de verre et á feuilles d'étains. L'air de la caisse métallique s'ozonise sous
}

l'influence des décharges qui se produisent entre les lames du condensateur.

Cette caisse est en comruunication par un ajutage métalliçue avec un robinet d'écoulement d'eau qui porte à son orifice un émulseur. Par suite d'un dispositif spécial, lorsqu'on ouvre le robinet, on met le contact dans le producteur d'ozone, l'eau en s'écoulant forme trompe et aspire l'ozone produit; le brassage s'opère dans l'émulseur et l'eau au sortir de l'appareil est complètement stérilisée.

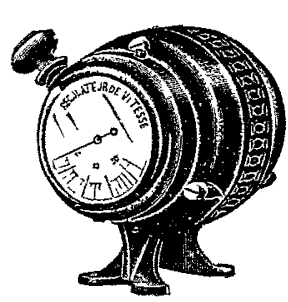

Pour utiliser le courant continu on adjoint à l'appareil un petit moleur actionnant un inverseur rotati?.

La Compagnie, qui exposail aussi plusieurs compteur's de differents syslèm,es, et divers apparcils de mesures, s'est vu décerner un troisième prix avec inédaille de bronze.

Détrartreur de tubes de chaudières - Les chaudièrea multitubulaires, dont remploi se génél'alise de plus en plus, ont le gros inconvénient de s'entartrer lapidement lor'sque les eaux d'alimentation sont calcaires. Pour enlever ce dépôt, qui obstrue en parlie les tubes, on emploie généralement des raclettes dont le maniement est dilficile. L'opération est par suite longue et peu elficace.

La Société pour le Travail électrique des Mélaux a présenté un détaıtreur électrique qui se composed'un moteur spécial de forme allongée $\mathrm{A}$, monté à l'extrémité d'un tube $\mathrm{B}$, qui lui sert de marche; il est, de plus, recouvert d'une enveloppe étanche. Le tout a un diamètre inférieur' a celui du tube à détartrer. L'arbre de linduit du moteur so prolonge à l'extérieur, et porte une boucle sur lacjuelle vient se monter le marteau détartreur. Ce marteau est constitué par une molette en acier, montée sur un arbre en fer et dont l'cxtrémité est recourbée en forme de clochct; c'est ce crochet que l'on assujettit à la boucle de l'arbre de linduit.

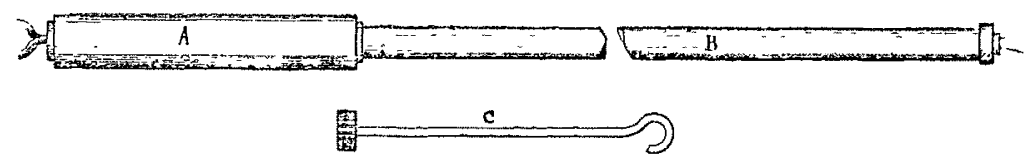

On introduit le tout dans le tube à neltoyer et l'on met le moteur en marche. Sous l'influence de la vitesse de rotalion, la molette vient frapper la paroi intéricure du tube et désagrège les dépôts calcaires que l'on évacue par un simple lavage. Les molettes étant folles sur leurs axes no peuvent apporter aucun dommage aux faisceaux tubulaires.

Cet appareil, après avoir été muni de molettes spéciales, rend les mêmes services pour les chaudières semi-tubulaires, car en débarrassant l'intérieur des tubes de la suic, il détache en mème temps, par les chocs rapides qu'il produit, le tartre qui recouvre la surface extérieure.

Les câbles de connexion du moteur à la source d'énergic passent à l'intérieur du tube d'acier et ne gênent en rien l'opérateur.

Le Jury a attribué à cet appareil un troisième prix avec médaille de bronze.

Pompes centrifuges électriques. - La Société des anciens établissements Luc-CourT et $\mathrm{C}^{1 e}$, exposait un groupe pompo centrifuge - moteur électrique monté sur même bảti. Un système de tuyauterie permetlait de prendre leau dans un baquet et de l'élever à une certaine hauteur, d'ıú ello retombait à son point de départ. On pouvait ainsi approximativernent se rendre compte de visu de la puissance du moteur et de la pompe.

Cette société exposait aussi divers petits moteurs à courant continu et à courant alternatif. L'un de ces dernier's 
actionnait une forte machins à coudre les cuirs, présentée par la maison IIurtu. Les sociétés Luc-CourT et HuRTu se sont vue décerner ex cequo un troisième prix avec médaille de bronze.

M. Plissonier, constructeur de machines agricoles, exposait également une pompe rotative actionnée par un

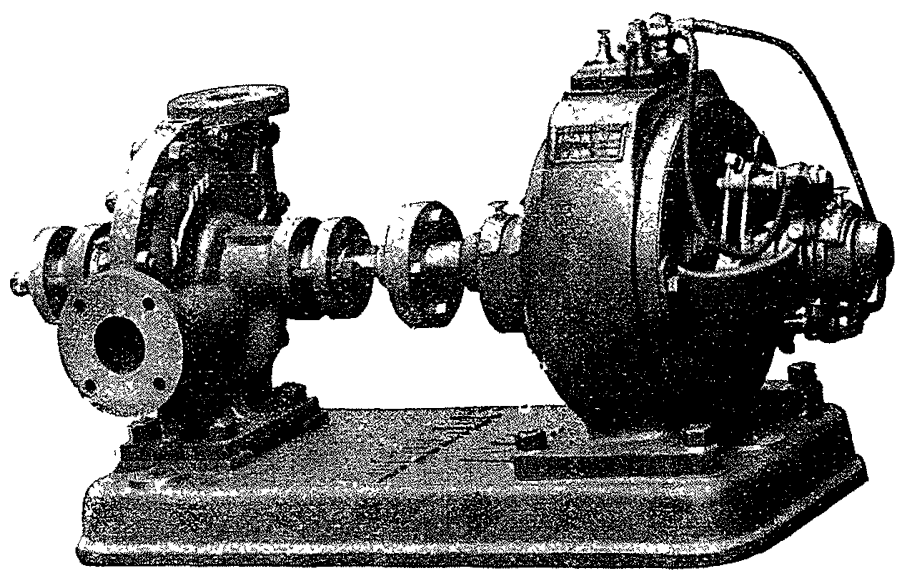

moleur électrique Limb. Ce groupe fonctionnait à côté du groupe Luc Court, et de la même façon, en puisant l eau dans le mème baquet.

M. Plissonier exposait aussi une écrémeuse, un concasseur de grains et un hâche-racines.

Dynamo à fer induit semi-fixe système Limb-Plasson.Les dynamos actuellement employées peuvent être classées en deux catégories: celles à induit avec fer et celles à induit sans fer. Les deux systèmes ont leurs avantages et leurs inconvénients. Dans le premier système, les pertes

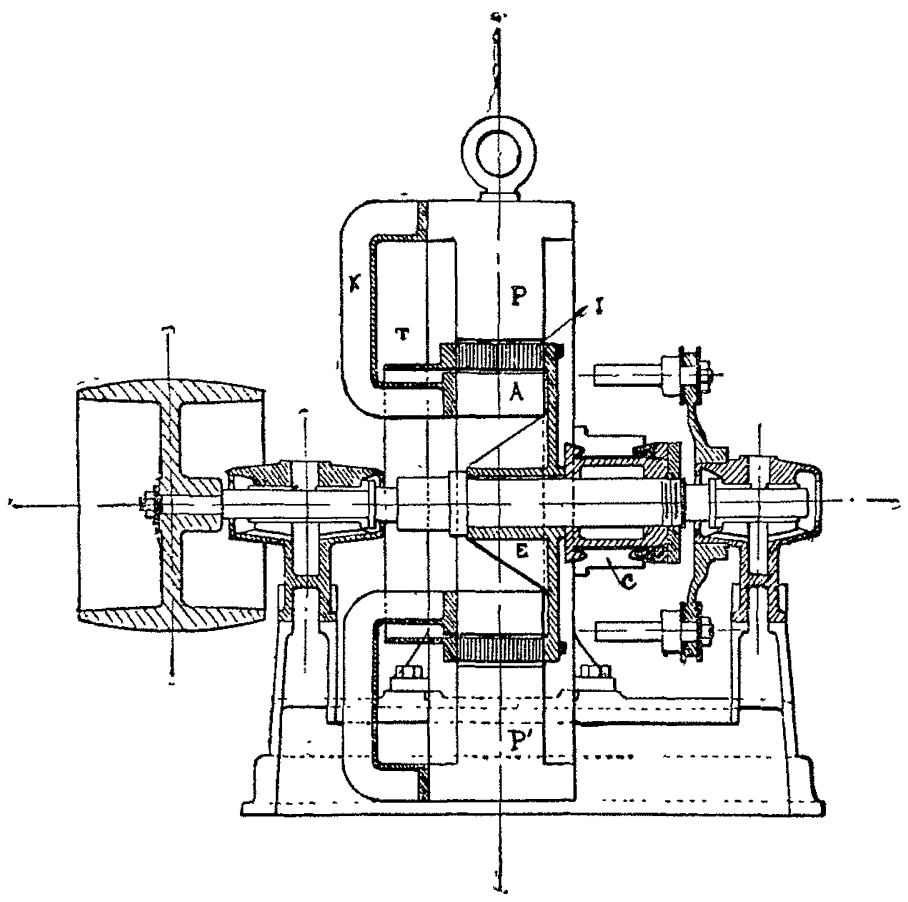

d'énergie par hystérésis et par courants de Foucault diminuent le rondement; de plus, la grande masse de fer qui tourne exige un axe de grosse section et des paliers en conséquence. Celles du second type sont peu robustes, à cause de la constitution de l'induit fait simplement de conducteurs agglomérés; de plus, elles exigent une grande excitation et un entrefer considélable ; aussi cə système est-il à peu près complètement abandonné, bien qu'il ait l'avantage d'annuler les pertes par hystérésis et par courants de Foucault.

La dynamo système Limb-Plasson réunit les avantages des deux types, tout en évitant une bonne partie de leurs inconvénients. Ce résultat a été obtenu en laissant fixcla plus grande partie du fer de l'induit, la partie mobile comprenant seulement celle nécessaire au logement des con. ducteurs de cet induit.

N'étant plus limité par l'hysstérésis, on peut augmenter l'induction magnétique, et par suite le nombre des ampères qui circulent dans linduit, sans craindre des réactions nuisibles. Pour cette raison, ces machines conviennent très bien pour les types multipolaires, comme les commutatrices, qui nécessitent un grand nombre de pôles si l'on veut éviter une trop grande vitesse angulaire.

L'induit élant très mince dans sa partie mobile présenle une grande surface relative au refroidissement. Il faut romarquer qu'il y a bien doux entrefers, mais leur valeur tolale est inférieure à celle de l'entrefer du type à induit sans fer.

Dans le type présenté, qui était de petites dimencions, les pôles inducteurs sont placés extérieurement, I est la partie moble du fer de l'induit monté en porte à faux sur une étoile en bronze clavetée sur l'arbre, P P' sont les pôles induzteurs, $\mathrm{K}$ une étoile nervurée réliant mécaniquement l'anneau A à la couronne du circuit inducteur. L'anneau A est un massif en fer qui complète le circuit magnélique et qui constitue la partie fixe de l'induit. Sur la partie mobile de l'induit se trouve un cylindre $\mathrm{T}$, en métal non magnétique et résistart, c'est sur ce tube que sont développées les connexions du tambour opposées au collec. teur C.

La maison Gindre Duchavany et $\mathrm{C}^{1 e}$ qui présentait un petit modèle de ce genre de machines, exposait aussi tout une série d'accumulateurs Mouterde de diverses dimensions $\left(^{*}\right)$. Elle s'est vu attribuer un $3^{\mathrm{e}}$ prix avec médaille de bronze et prime de 50 francs.

Moteurs divers. - A M. BONNIER, qui exposait divers petits moteurs électriques, a décerné un premier prix

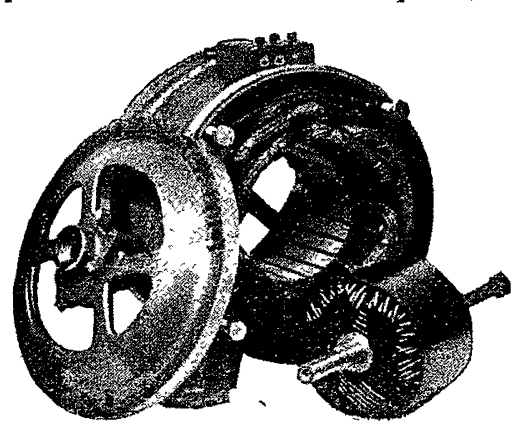
avec médaille de vermeil. L'un de ses moteurs ac. tionnait la machine à imprimer de M. Rua, un aulre le pétrin mécanique de $M$. Bérutti. On voyait également dans son stand quelques machines à coudre, et un tour à métaux actionné par un moteur triphasé à très faible vitesse.

La maison RousSELle et TOURNAIRE exposait toute une série de moteurs à courant continu et à courant alternatif; on y voyait plusieurs ventilateurs d'appartements, marchant sur 110 volts, et montés sur des supports à genouillère permettant leur orientation dans n'importe quel sens; puis des petites machines-outils utilisant directement la force du moteur sur son arbre.

Dans cette dernière catégorie, on remarquait une perceuse fixe pour établi se composant d'un moteur à courant continu à axe vertical, et d'une puissance de $1 / 10$ de cheval. Un train d'engr'enages réduit la vitesse du moteur. Le tout est logé dans un même carter, monté sur un bâti vertical dont le socle porte un arbre sur lequel est calé un pignon denté; celui-ci engrène avec une crémaillère qui supporle le plateau porte-pièce. La commande du plateau se fait par un petit levier qui actionne l'interrupteur et est placé à l'extérieur du bâti.

On vnyait aussi une perceuse portative remarguable par son extrême légèreté. Le bâti, qui forme les deux poignées, est en bronze d'aluminium. L'interrupteur, comme dans l'autre modèle, est placé dans la poignée.

( $\left.{ }^{\star}\right)$ Pour la description de ces accumulateurs, voir 'e dernier numéro de La Houille Blanche. 
On y voyait encore deux moteurs à courant continu dont l'arbre se prolonge à l'extérieur et est destiné à recevoir des meules émeri, des tampons à polir, des brosses, etc.

Cette maison a obtenu un troisième prix avec médaille de bronze.

La maison CADIoT présentait une petite machine univer. selle très pratique. Le moteur, à courant alternatif monophasé, est d'une puissance de $1 / 6^{2}$ de cheval, il est supporté parun bâti et est complètement enveloppé d'un carter sphérique. Sur l'axe du moleur, on peut adapter indifféremment une petite scie à métaux, une mêche à percer, une meule émeri ou bien encore des tampons à polir. Les carters servent à protéger ces divers outils et à recevoir les limailles, ils sont aussi destinés à servir de supports de pièces pour la meule et pour la scie.

La maison DuKARD présentait des appareils de chauffage, chauffe-pieds pour lram ways et chauffe-plats. Les chauffepieds de tramways servent pour 24 vorageurs et sont composés de cinq plaques chauffantes; la dépense en électricité est de 1100 vatts, soit 45 watts par voyageur. Le chauffe-plat est à une seule plaque chauffante et consomme à peu près 1 hectowatt; il fonctionne sous le courant de 120 volts. Contrairement aux radiateurs ordinaires, les plaques chauffantes de ces appareils ne comportent pas de matière isolante, cause d'absorption de chaleur.

La Société française d'électricité A.E.G. présentait, dans son stand, une série de moteurs à courant alternatif de faible puissance, pour machines à coudre, machines-outils, machines pour dentistes, etc. Ces moteul's sont remarquables par la robustesse de leur construction. Des flasques portepaliers protègent les enroulements contre toute at teinte extérieure. La partie tournante est constituée soit par un induit à court-circuit, soit par induit qui aboutit à 3 bagues permettunt l'adjonction de résistance dans le circuit induit. Ces moteurs, par suite de l'absolue symétrie de toutes les pièces, peuvent se placer indifféremment par terre, se fixer au plafond ou contre un mur.

Outre cette série de moteurs, on remarquait encore une petite perceuse pour établi, du même modèle que celle décrite plus haut, et une perceuse portative avec réducteur de vitesse. Il y avait également un moteur de machine à coudre monté sur glissière pour permetlre de tendre la courroie; le démarrage se fait au moyen d'un rhéostat spécial commandé par la pédale de la machine.

Le jury a attribué à cette société un troisième prix avec médaille de bronze.

Même récompense a été décérnée à la Société des tondeuses électriques, qui, outre des tondeuses, exposait aussi divers petits moteurs.

\section{La Distribution de l'Énergie Électrique dans la Ville de Grenoble}

\footnotetext{
Conférence taite le 28 mai I906, devant la Soctété pour le développement de l'Enseignement technique prés l'Université de Grenoble, nar M.F. JENnY, Ingenieur, chef du service electrique de la ville de Grenoble.

Sur la demande de M. Barbillon, l'éminent directeur de I'Institut Electrotechnique de Grenoble et du Bureau de contrôle municipal, je me fais un véritable plaisir de venir vous exposer en quelques mots la façon dont notre ville est alimentée en énergie électrique.

Je passeraí rapidement sur la première partie de cette installation, comprenant l'usine génératrice et la ligne d'adduction à très haut voltage, qui, assurément, présente un très grand intérêt, mais qui ne dépend pas de mon service.
}

L'exploitation de l'éclairage électrique de la cité grenobloise est entièrement municipale, fait qui se rencontre très rarement en France. L'énergie est fournie à la ville, aux bornes secondaires des transformateurs, a la tension de 5 ooo volts, par la Société Electro-chimique de la Romanche qui a la concession totale et unique pour une durée de 45 ans.

\section{USINE GÉNÉRATRICE}

L'usine génératrice est située à Livet, petit village adossé aux pieds des rochers gigantesques des massifs du Taillefer et de Belledonne, et traversé par un torrent impétueux, " la Romanche ", affluent du Drac, et qui prend sa source dans l'Oisans. C'est ce torrent qui fournit l'énergie nécessaire au fonctionnement des turbines de l'usine de la Société Electro-chimique de la Romanche. L'eau est captée au pont de l'Aveynat, à 2 kilomètres environ en amont de Livet, et est amenée à la chambre d'eau, située au-dessus de l'usine, par une galerie souterraine de section sensiblement circulaire, de $3 \mathrm{~m}$. 75 de diamètre. De là, l'eau est conduite sous pression dans le collecteur par une conduite forcée verticale en tôle d'acier, de $2 \mathrm{~m}$. 50 de diamètre, et placée dans un puits foré dans le rocher. La hauteur de la chute est de 60 mètres environ.

Les turbines sont capables de développer chacune une puissance normale de 25 oo chevaux à la vitesse de 375 tours à la minute, mais, en réalité, elles peuvent fournir une puissance un peu plus élevée, afin de répondre dans une certaine mesure à la surcharge que les alternateurs peuvent supporter. Elles ont été construites par la Maison A. et $\mathrm{H}$. Bouvier, de Grenoble. Chaque turbine commande, au moyen d'un accnuplement direct, un alternateur de 2500 kilovoltampères, pour $\cos \varphi=0,7$, produisant du courant triphasé à 50 périodes sous 3 ooo volts composés.

Le courant engendré à cette tension, après avoir passé par un tableau renfermant les appareils de sécurité et de manœuvre les plus modernes, est conduit dans un transformateur de même puissance que l'alternateur, et est transformé à la tension de 26 ooo volts.

Il est intéressant de remarquer que le tableau dont on vient de parler permet le couplage de n'importe quelle machine sur un transformateur quelconque.

Le matériel électrique a été fourni et installé par la maison Brown et Boveri de Baden (Suisse).

Le courant à 26 ooo volts, venant des transformateurs, est dirigé ensuite sur un tableau de couplage de lignes, en ciment armé, d'où partent 2 lignes triphasées à 3 fils.

Chaque ligne passe par un interrupteur automatique à huile, à action différée, et par un parafoudre dont nous donnerons une description détaillée dans l'étude de la sousstation de Grenoble.

Notons encore, avant de quitter l'usine, qu'elle renferme 3 groupes analogues à celui que nous venons de décrire, et que les moteurs hydrauliques sont munis d'un régulateur d'un nouveau système construit par la maison A. et $\mathrm{H}$. Bouvier de Grenoble.

Ce système présente cette particularité que, pour la première fois, il donne la solution du règlage simultané de toutes les unités d'une station centrale au moyen d'un seul pendule. A ce sujet, il est bon de rappeler que la Société Internationale des Electriciens s'est vivement préocupée de cette question, et que, dans son bulletin d'avril 1902 , certains de ses membres émettaient l'opinion que la régularisation par un seul régulateur conduirait à une solution parfaite du problème, mais que cétte solution semblait très difficile à 